\title{
CHARACTER AND DIMENSION FORMULAE FOR QUEER LIE SUPERALGEBRA
}

\author{
YUCAI SU AND R. B. ZHANG
}

\begin{abstract}
Closed formulae are constructed for the characters and dimensions of the finite dimensional simple modules of the queer Lie superalgebra $\mathfrak{q}(n)$. This is achieved by refining Brundan's algorithm for computing simple $\mathfrak{q}(n)$-characters.
\end{abstract}

\section{INTRODUCTION}

There has been considerable progress in the representation theory [13] of Lie superalgebras [12, 19] in recent years. In 11, Brundan reformulated Serganova's KazhdanLusztig approach [17] to the parabolic category $\mathcal{O}$ of the general linear superalgebra $\mathfrak{g l}(m \mid n)$ using quantum group techniques, obtaining a very practicable algorithm for computing the generalized Kazhdan-Lusztig polynomials. This enabled him to prove the conjecture of [26] on the composition factors of Kac modules. By using this algorithm, the present authors [21] derived closed character and dimension formulae for the finite dimensional simple $\mathfrak{g l}(m \mid n)$-modules. An algorithm was developed by Serganova and Gruson [18, 10], which enables one to compute the characters of the finite dimensional simple modules for the orthosymplectic Lie superalgebras $\mathfrak{o r p}(m \mid 2 n)$. In the special case of $\mathfrak{o s p}(m \mid 2)$, the characters and dimensions of the simple modules were worked out explicitly [23].

Properties of the parabolic categories $\mathcal{O}$ for simple Lie superalgebras (relative to the maximal parabolic subalgebra with a purely even Levi subalgebra) have also been investigated extensively. It was discovered in [8, 9, that the representation theory of the classical Lie superalgebras $\mathfrak{g l}(m \mid n)$ and $\mathfrak{o} \mathfrak{s p}(m \mid 2 n)$ was related to that of infinite dimensional classical Lie algebras in some precise way. This led to the super duality conjecture in [7], which was proven in [5, 4] for $\mathfrak{g l}(m \mid \infty)$ and generalised to $\mathfrak{o s p}(m \mid \infty)$ in [6]. A Jantzen type filtration for parabolic Verma modules of the type I and exceptional Lie superalgebras was developed [22, 24]. It was shown that the layers of the Jantzen type filtration are semi-simple and the multiplicities of their composition factors are determined by the coefficients of the inverse of Serganova's generalised Kazhdan-Lusztig polynomials.

Penkov and Serganova [16] developed an algorithm for computing the characters of finite dimensional simple modules for the queer Lie superalgebra $\mathfrak{q}(n)$. They used a super analogue of the Bott-Borel-Weil theory [14, 15] to realise $\mathfrak{q}(n)$-representations on cohomology groups of vector bundles on super flag varieties of the corresponding supergroup $Q_{n}$. Their algorithm enables one to understand composition factors of the cohomology groups combinatorially. Brundan provided an important new input into the problem [2] by translating it to a problem about some canonical bases of the

2010 Mathematics Subject Classification. Primary 17B10.

This work was supported by the Australian Research Council (grant no. DP0986551), NSF of China (grant no. 10825101), SMSTC (grant no. 12XD1405000) and the Fundamental Research Funds for the Central Universities of China. 
quantum group associated with the infinite dimensional Lie algebra of type $\mathfrak{b}_{\infty}$. By working out the combinatorics of the canonical bases, he obtained a more efficient algorithm for determining the composition factors.

The algorithms are sufficient in some situations, especially when the problems concerned require only knowledge of characters of specific simple $\mathfrak{q}(n)$-modules. However, for some problems, e.g., if one wants to determine the dimensions of finite simple modules in a uniform way, the algorithms are not adequate and a closed formula for the characters will be needed. At any rate, a character formula is more desirable.

In this paper, we develop a closed formula of Weyl-Kac type for the characters of the finite dimensional simple modules for the queer Lie superalgebra $\mathfrak{q}(n)$, and derive from it a formula for the dimensions of the simple modules. The character formula is obtained by using a refined version of the algorithm of [2]. The main results of the paper are presented in Section 4. In particular, Theorem 4.8 gives the character and dimension formulae, and Corollary 4.9 rewrites the formulae in more convenient terms. This work is a continuation of [21] but for $\mathfrak{q}(n)$.

The organization of the paper is as follows. In Section 2 we present some background material on $\mathfrak{q}(n)$, which will be used throughout the paper. In Section 3 we develop some combinatorics for the Euler modules. [2, Main Theorem] is reformulated in terms of weight diagrams, providing an algorithm which is more effective for what we are trying to do here. Section 4 contains the main results.

\section{Preliminaries}

2.1. The queer Lie superalgebra $\mathfrak{q}(n)$. A super vector space over $\mathbb{C}$ is a $\mathbb{Z}_{2^{-}}$ graded vector space $V=V_{\overline{0}} \oplus V_{\overline{1}}$, where $\mathbb{Z}_{2}=\{\overline{0}, \overline{1}\}$. Denoted by $[v]$ the parity of $v$ which is either $\overline{0}$ or $\overline{1}$ according to whether $v \in V_{\overline{0}}$ or $v \in V_{\overline{1}}$. A vector $v$ is even if $[v]=\overline{0}$ and odd otherwise. For two super vector spaces $V$ and $W$, a linear map $f: V \rightarrow W$ is homogeneous of degree $d \in \mathbb{Z}_{2}$ if $f\left(V_{a}\right) \subset W_{a+d}$ for $a \in \mathbb{Z}_{2}$. Denote $\operatorname{Hom}(V, W)_{d}=\{f: V \rightarrow W \mid f$ is homogeneous of degree $d\}$. Then $\operatorname{Hom}(V, W)=\operatorname{Hom}(V, W)_{\overline{0}} \oplus \operatorname{Hom}(V, W)_{\overline{1}}$ is a super vector space. The dual super vector space of $V$ is defined to be $V^{*}=\operatorname{Hom}(V, \mathbb{C})$. For homogeneous linear maps $f_{1}: V_{1} \rightarrow W_{1}$ and $f_{2}: V_{2} \rightarrow W_{2}$, the tensor product $f_{1} \otimes f_{2}: V_{1} \otimes V_{2} \rightarrow W_{1} \otimes W_{2}$ is a homogeneous linear map defined by $\left(f_{1} \otimes f_{2}\right)\left(v_{1} \otimes v_{2}\right)=(-1)^{\left[f_{2}\right]\left[v_{1}\right]} f_{1}\left(v_{1}\right) \otimes f_{2}\left(v_{2}\right)$. The super vector spaces and the homogeneous mappings above define a category, which is not abelian. However, if we restrict the Hom-sets to the even mappings, we obtain an abelian category, denoted by $\mathfrak{O}$. Denote by $\Pi: \mathfrak{O} \rightarrow \mathfrak{O}$ the parity change functor, defined as follows: if $V \in \mathfrak{O}$ we set $(\Pi(V))_{d}=V_{d+1}$ for $d \in \mathbb{Z}_{2}$, and if $f: V \rightarrow W$ in $\mathfrak{O}$ we set $\Pi(f)=f$ as mappings. We denote by $\Pi(\mathbb{C})$ the odd super vector space of dimension one.

For any positive integer $n$, let $\mathfrak{g}=\mathfrak{q}(n)$ denote the queer Lie superalgebra consisting of the block matrices of the form $\left(\begin{array}{ll}A & B \\ B & A\end{array}\right)$ for $A, B \in g l_{n}(\mathbb{C})$. The even (resp., odd) subspace $\mathfrak{g}_{\overline{0}}$ (resp., $\mathfrak{g}_{\overline{1}}$ ) of $\mathfrak{g}$ consists of block matrices with $B=0$ (resp., $A=0$ ). Let

$$
e_{i j}^{\overline{0}}=\left(\begin{array}{cc}
E_{i j} & 0 \\
0 & E_{i j}
\end{array}\right), \quad e_{i j}^{\overline{1}}=\left(\begin{array}{cc}
0 & E_{i j} \\
E_{i j} & 0
\end{array}\right) \text { for } 1 \leq i, j \leq n,
$$

denote respectively the basis elements of $\mathfrak{g}_{\overline{0}}$ and $\mathfrak{g}_{\overline{1}}$, where $E_{i j} \in g l_{n}(\mathbb{C})$ is the matrix unit with 1 at position $(i, j)$ and 0 elsewhere.

Then the supercommutator is defined as follows:

$$
\left[e_{i j}^{s}, e_{k l}^{t}\right]=\delta_{j k} e_{i l}^{s+t}-(-1)^{s t} \delta_{i l} e_{k j}^{s+t},
$$


where $s, t \in \mathbb{Z}_{2}$ and $1 \leq i, j \leq n$. Take $\mathfrak{h}=\operatorname{span}_{\mathbb{C}}\left\{e_{i i}^{s}, \mid s \in \mathbb{Z}_{2}, 1 \leq i \leq n\right\}$ to be the Cartan Lie subsuperalgebra. Then $\operatorname{sdim}(\mathfrak{h})=n+n \bar{\varepsilon}$ and $\mathfrak{h}_{\overline{0}}$ is a Cartan subalgebra of $g l_{n}(\mathbb{C})$. The elements $H_{i}=e_{i i}^{\overline{0}}$ (resp., $\hat{H}_{i}=e_{i i}^{\overline{1}}$ ) for $1 \leq i \leq n$ form a basis in $\mathfrak{h}_{\overline{0}}$ (resp., $\left.\mathfrak{h}_{\overline{1}}\right)$. Let $\varepsilon_{i}, 1 \leq i \leq n$, be the basis of $\mathfrak{h}_{\overline{0}}^{*}$ dual to the basis $\left\{H_{i} \mid 1 \leq i \leq n\right\}$ of $\mathfrak{h}_{\overline{0}}$, that is, $e_{i}\left(H_{j}\right)=\delta_{i j}$ for all $i, j$. Then $\mathfrak{h}_{0}^{*}$ is equipped with the bilinear form $(\cdot, \cdot)$ defined by

$$
\left(\varepsilon_{i}, \varepsilon_{j}\right)=\delta_{i j}, \quad \forall i, j .
$$

We have a root space decomposition of $\mathfrak{g}$ with respect to $\mathfrak{h}_{\overline{0}}$, given by

$$
\mathfrak{g}=\mathfrak{h} \bigoplus\left(\bigoplus_{\alpha \in \Delta_{\overline{0}}} \mathfrak{g}^{\alpha}\right) \bigoplus\left(\bigoplus_{\alpha \in \Delta_{\overline{1}}} \mathfrak{g}^{\alpha}\right)
$$

where $\Delta_{\overline{0}}=\left\{\varepsilon_{i}-\varepsilon_{j} \mid 1 \leq i, j \leq n, i \neq j\right\}$ is the set of even roots, and $\Delta_{\overline{1}}$ is the set of odd roots, which is isomorphic to $\Delta_{\overline{0}}$ as set. Let $\Delta=\Delta_{\overline{0}} \sqcup_{\overline{1}}$ (disjoint union). We have $\mathfrak{g}^{\alpha}=\mathbb{C} e_{i j}^{s}$ if $\alpha=\varepsilon_{i}-\varepsilon_{j} \in \Delta_{s}, s \in \mathbb{Z}_{2}$. Then $\Delta_{\overline{0}}$ is the root system of $\mathfrak{g}_{\overline{0}}=g l_{n}(\mathbb{C})$. The positive root system is $\Delta^{+}=\Delta_{\overline{0}}^{+} \sqcup \Delta_{\overline{1}}^{+}$with $\Delta_{\overline{0}}^{+}=\left\{\varepsilon_{i}-\varepsilon_{j} \mid 1 \leq i<j \leq n\right\}$ and similarly for $\Delta_{\overline{1}}^{+}$. The simple root system is $\Pi^{+}=\Pi_{\overline{0}}^{+} \sqcup \Pi_{\overline{1}}^{+}$with $\Pi_{\overline{0}}^{+}=\left\{\varepsilon_{i}-\varepsilon_{i+1} \mid 1 \leq i \leq n-1\right\}$ and similarly for $\Pi_{\overline{1}}^{+}$. Set

$$
\rho_{\overline{0}}=\frac{1}{2} \sum_{\alpha \in \Delta_{\overline{0}}^{+}} \alpha=\frac{1}{2}(n-1, n-3, \ldots, 1-n),
$$

and $\rho_{\overline{1}}=\frac{1}{2} \sum_{\alpha \in \Delta_{\overline{1}}^{+}} \alpha=\rho_{\overline{0}}$ and $\rho=\rho_{\overline{0}}-\rho_{\overline{1}}=0$. Note that $\rho_{\overline{0}}$ corresponds to half of the sum of the positive roots of the Lie algebra $\mathfrak{g}_{\overline{0}} \cong g l_{n}(\mathbb{C})$.

The Borel subsuperalgebra of $\mathfrak{g}$ is $\mathfrak{b}=\mathfrak{h} \oplus \mathfrak{n}^{+}$, where $\mathfrak{n}^{+}=\oplus_{\alpha \in \Delta^{+}} \mathfrak{g}^{\alpha}$. Take any parabolic subalgebra $\mathfrak{p}_{\overline{0}} \supseteq \mathfrak{b}_{\overline{0}}$ of $\mathfrak{g}_{\overline{0}} \cong g l_{n}(\mathbb{C})$, and let $\Delta\left(\mathfrak{p}_{\overline{0}}\right)$ be the set of roots of $\mathfrak{p}_{\overline{0}}$, that is, $\alpha \in \Delta\left(\mathfrak{p}_{\overline{0}}\right)$ if and only if $\mathfrak{g}^{\alpha} \subset \mathfrak{p}_{\overline{0}}$. Let $\Delta\left(\mathfrak{p}_{\overline{1}}\right)$ be a subset of $\Delta_{\overline{1}}$ which is isomorphic to $\Delta\left(\mathfrak{p}_{\overline{0}}\right)$, and set $\Delta(\mathfrak{p})=\Delta\left(\mathfrak{p}_{\overline{0}}\right) \sqcup \Delta\left(\mathfrak{p}_{\overline{1}}\right)$ (disjoint union). Then $\mathfrak{p}=\mathfrak{h} \oplus\left(\bigoplus_{\alpha \in \Delta(\mathfrak{p})} \mathfrak{g}^{\alpha}\right)$ is a parabolic subalgebra containing the Borel subalgebra $\mathfrak{b}$. We may also define the lower triangular Borel subalgebra $\overline{\mathfrak{b}}$ and parabolicc subalgebras $\overline{\mathfrak{p}} \supset \overline{\mathfrak{b}}$.

2.2. Integral dominant weights. We will express a weight $\lambda \in \mathfrak{h}_{\overline{0}}^{*}$ in term of its coordinate relative to the basis $\left(\varepsilon_{1}, \ldots, \varepsilon_{n}\right)$ as $\lambda=\sum_{i=1}^{n} \lambda_{i} \varepsilon_{i}$ and simply write

$$
\lambda=\left(\lambda_{1}, \ldots, \lambda_{n}\right) \in \mathbb{C}^{n} .
$$

Introduce the following integers

$$
\begin{aligned}
& \left.z(\lambda)=\#\left\{i \mid \lambda_{i}=0\right\} \quad \text { (the number of zero entries of } \lambda\right), \\
& \bar{z}(\lambda)=0 \text { if } z(\lambda) \text { is even or } 1 \text { else (the parity of } z(\lambda)), \\
& h(\lambda)=n-z(\lambda) \quad \text { (the number of nonzero entries of } \lambda) .
\end{aligned}
$$

A weight $\lambda$ is called integral if $\lambda \in \mathbb{Z}^{n}$, and integral dominant if $\lambda \in \mathbb{Z}_{++}^{n}$, where

$$
\mathbb{Z}_{++}^{n}=\left\{\lambda \in \mathbb{Z}^{n} \mid \lambda_{1} \geq \cdots \geq \lambda_{n} \text { and } \lambda_{i}=\lambda_{i+1} \text { implies } \lambda_{i}=0 \text { for any } i\right\} .
$$

Let $W \cong \operatorname{Sym}_{n}$ be the Weyl group of $\Delta_{\overline{0}}$, which acts on both $\mathfrak{h}_{\overline{0}}$ and $\mathfrak{h}_{\overline{0}}^{*}$ in the usual way. As $\rho=0$, the dot action of $W$ coincides with the usual action. Define a total order on $\mathbb{Z}_{++}^{n}$ lexicographically, namely

$$
\lambda<\mu \Longleftrightarrow \text { for the first } p \text { with } \mu_{p} \neq \lambda_{p} \text {, we have } \mu_{p}<\lambda_{p} .
$$


Definition 2.1. An integral weight $\lambda$ is called

(1) regular or non-vanishing (in sense of [11, 25]) if it is $W$-conjugate to an integral dominant weight (which is denoted by $\lambda^{+}$throughout the paper);

(2) vanishing otherwise (since the right-hand side of (4.25) vanishes in this case).

Obviously, $\lambda$ is regular if and only if

any nonzero number appears at most once as an entry of $\lambda$.

Let $\lambda$ in (2.6) be a regular weight. A positive root $\gamma=\varepsilon_{i}-\varepsilon_{j}$ is an atypical root of $\lambda$ if $\lambda_{i}=-\lambda_{j}$. In case $\left\lfloor\frac{z(\lambda)}{2}\right\rfloor>0$ (where $\lfloor a\rfloor$ denotes the integer part of $a$ ), we always take $\left\lfloor\frac{z(\lambda)}{2}\right\rfloor$ atypical roots $\gamma_{p}=\varepsilon_{m_{p}}-\varepsilon_{n_{p}}, p=1, \ldots,\left\lfloor\frac{z(\lambda)}{2}\right\rfloor$, to satisfy

$$
\lambda_{m_{p}}=\lambda_{n_{p}}=0, \quad m_{\left\lfloor\frac{z(\lambda)}{2}\right\rfloor}<\cdots<m_{2}<m_{1}<n_{1}<n_{1}<\cdots<n_{\left\lfloor\frac{z(\lambda)}{2}\right\rfloor} .
$$

Furthermore, if $\bar{z}(\lambda)=1$, we choose $m_{1}, n_{1}$ to be the indices such that the extra zero entry lies in between, i.e., there exists a unique $k_{0}$ with $m_{1}<k_{0}<n_{1}$ and $\lambda_{k_{0}}=0$. Denote by $\Gamma_{\lambda}$ the set of atypical roots of $\lambda(\mathrm{cf}$. (2.14) and (2.13) $)$ :

$$
\Gamma_{\lambda}=\left\{\varepsilon_{i}-\varepsilon_{j} \mid \text { either } \lambda_{i}=-\lambda_{j} \neq 0 \text {, or else } i=m_{p}, j=n_{p} \text { for some } p\right\} \text {. }
$$

Set $r=\# \Gamma_{\lambda}$. We also denote $\# \lambda=r$, called the degree of atypicality of $\lambda$. A weight $\lambda$ is called

- typical if $r=0$;

- atypical if $r>0$ (in this case $\lambda$ is also called an $r$-fold atypical weight).

When $\lambda$ is integral dominant, we always arrange the atypical roots of $\lambda$ as $\gamma_{p}=\varepsilon_{m_{p}}-\varepsilon_{n_{p}}$ for $1 \leq p \leq r$ with $m_{p}<m_{p+1}<n_{p+1}<n_{p}$ for $1 \leq p<r$. Thus,

$$
\Gamma_{\lambda}=\left\{\gamma_{1}<\gamma_{2}<\cdots<\gamma_{r}\right\}
$$

by the order (2.9), and $\lambda$ has the following form:

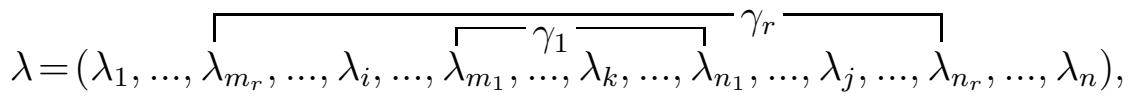

2.3. Formal characters. Let $V=\oplus_{\lambda \in \mathfrak{h}} V_{\lambda}$ be a weight module over $\mathfrak{g}$, where

$$
V_{\lambda}=\left\{v \in V \mid h v=\lambda(h) v, \forall h \in \mathfrak{h}_{\overline{0}}\right\} \text { with } \operatorname{dim} V_{\lambda}<\infty,
$$

is the weight space of weight $\lambda$. We denote by $\operatorname{ch} V=\sum_{\lambda \in \mathfrak{h}_{0}^{*}}\left(\operatorname{dim} V_{\lambda}\right) e^{\lambda}$ its character.

Given $\lambda \in \mathbb{Z}_{++}^{n}$, there is a unique (up to isomorphism) finite dimensional simple $\mathfrak{g}$-module $L(\lambda)$ with highest weight $\lambda$ such that $\operatorname{dim}\left(\operatorname{End}_{\mathfrak{g}} L(\lambda)\right)=1$ or 2 . We say that $L(\lambda)$ is of type $M$ in the former case, and type $Q$ in the later case. For example, the natural representation $V=L\left(\varepsilon_{1}\right)$ of $\mathfrak{g}$ is of type $\mathrm{Q}$, as there exists an odd automorphism $\theta: v_{i} \mapsto v_{i+n}$ and $v_{i+n} \mapsto-v_{i}$ for $1 \leq i \leq n$, where $\left\{v_{i} \mid 1 \leq i \leq 2 n\right\}$ is the natural basis of $V$. In general, $L(\lambda)$ is of type $\mathrm{M}$ if $h(\lambda)$ is even, and of type Q otherwise.

For each $\lambda \in \mathbb{Z}^{n}$, there exists a unique simple $\mathfrak{h}$-module $\mathfrak{u}(\lambda)$. Each $e_{i i}^{(0)}$ acts on $\mathfrak{u}(\lambda)$ by $\lambda_{i} \operatorname{id}_{\mathfrak{u}(\lambda)}$, thus it follows from $\left[e_{i i}^{(1)}, e_{j j}^{(1)}\right]=2 \delta_{i j} e_{i i}^{(0)}$ for all $i, j$ that the actions of all $e_{i i}^{(1)}$ on $\mathfrak{u}(\lambda)$ generate an irreducible representation of the Clifford algebra of degree $h(\lambda)$. Hence $\operatorname{dim} \mathfrak{u}(\lambda)=2^{\left\lfloor\frac{h(\lambda)+1}{2}\right\rfloor}$ and the character of $\mathfrak{u}(\lambda)$ is given by $2^{\left\lfloor\frac{h(\lambda)+1}{2}\right\rfloor} e^{\lambda}$.

Denote by $G=Q_{n}$ the supergroup with Lie superalgebra $\mathfrak{q}(n)$. Let $\bar{B}$ be the lower triangular Borel subgroup of $Q_{n}$ with Lie superalgebra $\overline{\mathfrak{b}}$, and let $\bar{P}(\lambda) \supseteq \bar{B}$ be the 
largest parabolic subgroup such that $\mathfrak{u}(\lambda)$ can be lifted to a $\bar{P}(\lambda)$-module. The sheaf cohomology groups of the associated super vector bundle $\mathcal{L}(\mathfrak{u}(\lambda))=Q_{n} \times{ }_{\bar{P}(\lambda)} \mathfrak{u}(\lambda)$,

$$
H^{i}(\lambda)=H^{i}(G / \bar{P}(\lambda), \mathcal{L}(\mathfrak{u}(\lambda))), \quad i=0,1, \ldots,
$$

admit natural $G$-actions. It is known that $H^{i}(\lambda)$ are finite dimensional for all $i$ and vanish for $i \gg 0$. In particular, $H^{0}(\lambda)$ contains a unique simple submodule $L(\lambda)$, and $\{L(\lambda)\}_{\lambda \in \mathbb{Z}_{++}^{n}}$ is the complete set of pairwise non-isomorphic simple $G$-modules. Here $L(\lambda)$ is the highest weight $\mathfrak{g}$-module with highest weight $\lambda$ relative to $\mathfrak{b}$ (not $\overline{\mathfrak{b}}$ ).

For any $\lambda \in \mathbb{Z}_{++}^{n}$, we define the virtual module $E(\lambda)$, the Euler module, by

$$
E(\lambda)=\sum_{i \geq 0}(-1)^{i} H^{i}(\lambda)
$$

which should be interpreted as an element in the Grothendieck group of the category of finite dimensional $\mathfrak{g}$-modules. The Euler character ch $E(\lambda)=\sum_{i \geq 0}(-1)^{i} \operatorname{ch} H^{i}(\lambda)$ is given by (cf. [14, 2, 3]) the formula $\operatorname{ch} E(\lambda)=2^{\left\lfloor\frac{h(\lambda)+1}{2}\right\rfloor} P_{\lambda}$ with

$$
\begin{aligned}
P_{\lambda} & :=\sum_{w \in \operatorname{Sym}_{n} / S_{\lambda}} w\left(e^{\lambda} \prod_{\substack{1 \leq i<j \leq n \\
\lambda_{i}>\lambda_{j}}} \frac{1+e^{\varepsilon_{j}-\varepsilon_{i}}}{1-e^{\varepsilon_{j}-\varepsilon_{i}}}\right) \\
& =\frac{1}{\# S_{\lambda}} \sum_{w \in \operatorname{Sym}_{n}} w\left(e^{\lambda} \prod_{\substack{1 \leq i<j \leq n \\
\lambda_{i}>\lambda_{j}}} \frac{1+e^{\varepsilon_{j}-\varepsilon_{i}}}{1-e^{\varepsilon_{j}-\varepsilon_{i}}}\right)
\end{aligned}
$$

being Schur's P-function, where $S_{\lambda}$ is the stabilizer of $\lambda$ in $\operatorname{Sym}_{n}$, \# $S_{\lambda}$ is the size of $S_{\lambda}$, and $\operatorname{Sym}_{n} / S_{\lambda}$ denotes the set of minimal length coset representatives.

\section{Combinatorics of Euler modules}

3.1. Weight diagrams. We shall follow [4, 10, 23] to express integral dominant weights by weight diagrams. Given any $\lambda \in \mathbb{Z}_{++}^{n}$, we let

$$
\begin{array}{ll}
S(\lambda)_{\mathrm{L}}=\left\{\lambda_{i}>0 \mid 1 \leq i \leq n\right\}, & S(\lambda)_{\mathrm{R}}=\left\{-\lambda_{i}>0 \mid 1 \leq i \leq n\right\}, \\
S(\lambda)=S(\lambda)_{\mathrm{L}} \cup S(\lambda)_{\mathrm{R}}, & S(\lambda)_{\mathrm{B}}=S(\lambda)_{\mathrm{L}} \cap S(\lambda)_{\mathrm{R}} .
\end{array}
$$

Definition 3.1. Any element $\lambda \in \mathbb{Z}_{++}^{n}$ can be expressed in a unique way by a weight diagram $D_{\lambda}$ (cf. (3.3) ), which is the positive half of the real line with vertices indexed by $\mathbb{N}=\{0,1,2, \ldots\}$ such that

- the vertex $i=0$ is associated with $\left\lfloor\frac{z(\lambda)}{2}\right\rfloor$ many $\times$ 's, and if $\bar{z}(\lambda)=1$ an additional symbol $\perp$ at the top;

- each vertex $i>0$ is associated with a unique symbol $D_{\lambda}^{i} \in\{\emptyset,<,>, \times\}$ according to the following rule: $D_{\lambda}^{i}$ is

$$
\begin{array}{ll}
\emptyset, & \text { if } i \notin S(\lambda), \\
<, & \text { if } i \in S(\lambda)_{\mathrm{R}} \backslash S(\lambda)_{\mathrm{B}}, \\
>, & \text { if } i \in S(\lambda)_{\mathrm{L}} \backslash S(\lambda)_{\mathrm{B}}, \\
\times, & \text { if } i \in S(\lambda)_{\mathrm{B}} .
\end{array}
$$

Numerate the $\times$ 's from bottom to top at vertex 0 and then from left to right by $1,2, \ldots, r$ if there are $r$ of them in total. 
Thus the degree $\# \lambda$ of atypicality of $\lambda$ is the number of $\times$ 's in the weight diagram $D_{\lambda}$. For example, if $\mathfrak{g}=\mathfrak{q}(16)$ and

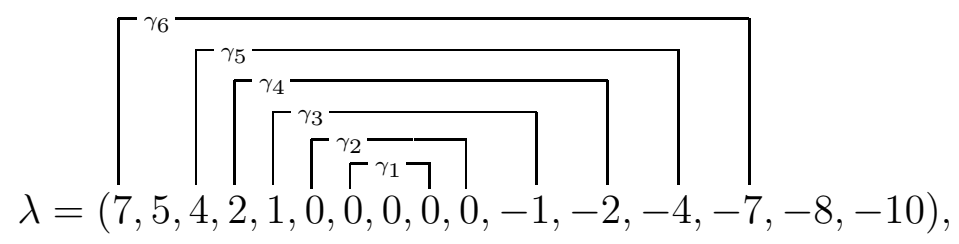

with the atypical roots

$$
\gamma_{1}=\varepsilon_{7}-\varepsilon_{9}, \quad \gamma_{2}=\varepsilon_{6}-\varepsilon_{10}, \quad \gamma_{3}=\varepsilon_{5}-\varepsilon_{11}, \quad \gamma_{4}=\varepsilon_{4}-\varepsilon_{12}, \quad \gamma_{5}=\varepsilon_{3}-\varepsilon_{13}, \quad \gamma_{6}=\varepsilon_{1}-\varepsilon_{14},
$$

then the weight diagram $D_{\lambda}$ is given by

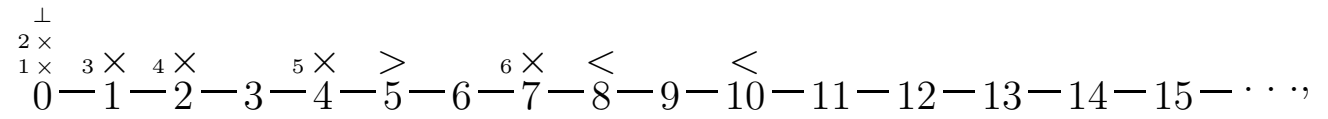

where, for simplicity, we have left out the symbol $D_{\lambda}^{i}=\emptyset$ from a vertex $i$ if $i \notin S(\lambda)$. In (3.3), the number of $\times$ 's is indeed $\# \lambda=6$.

3.2. Raising operators. Given any two vertices $s, t$ with $s \leq t$ in a weight diagram $D_{\lambda}$, we define the distance $d_{\lambda}(s, t)$ from $s$ to $t$ to be the number of $\emptyset$ 's minus the number of $\times$ 's strictly between these vertices. Note that the "distance" can be negative, and we remark that $d_{\lambda}(s, t)$ is the negative of the length $\ell_{\lambda}(s, t)$ defined in [23].

Suppose $\# \lambda=r$, then the $\times$ 's in $D_{\lambda}$ are labelled by $1, \ldots, r$. We denote by $x_{i}$ the vertex where the $i$-th $\times$ sits. For convenience, we denote $x_{0}=0$ (and imagine there is a 0 -th $\times$ sitting on the bottom at vertex 0 ).

Definition 3.2. (1) Given an $i$ such that $0 \leq i \leq r$ and any vertex $t>0$, we define the length $\ell_{\lambda}(i, t)$ from the $i$-th $\times$ to the vertex $t$ by

- $\ell_{\lambda}(i, t)=d_{\lambda}\left(x_{i}, t\right)$ if $x_{i}>0$, and

- $\ell_{\lambda}(i, t)=d_{\lambda}\left(x_{i}, t\right)-2\left(\left\lfloor\frac{z(\lambda)}{2}\right\rfloor-i\right)-\sharp(\perp)$ if $x_{i}=0$, where $\sharp(\perp)=0$ or 1 is the number of $\perp$ at vertex 0 .

(2) A right move (or raising operator) on $D_{\lambda}$ is to move to the right a $\times$, say the $i$-th one $(1 \leq i \leq r)$, to the first empty vertex $t$ (vertex with the symbol $\emptyset$ ) that meets the conditions $\ell_{\lambda}(i, t)=0$ and $\ell_{\lambda}(i, s)<0$ for all vertices $s$ satisfying $x_{i}<s<t$. We denote this right move by $R_{i}(\lambda)$. We also denote $k_{i}=t-x_{i}$, then we obtain $\left(k_{1}, \ldots, k_{r}\right)$, which will be referred to as the $r$-tuple of positive integers associated to $\lambda$.

(3) Given an element $\theta=\left(\theta_{1}, \ldots, \theta_{r}\right) \in\{0,1\}^{r}$, we set $|\theta|=\sum_{i=1}^{r} \theta_{i}$ and let $\theta_{i_{1}}, \ldots, \theta_{i_{|\theta|}}$ with $1 \leq i_{1}<\cdots<i_{|\theta|} \leq r$ be the nonzero entries. Associate to $\theta$ a unique right path (or raising operator) $R_{\theta}(\lambda)$ which is the collection of the $|\theta|$ right moves $R_{i_{1}}(\lambda), \ldots, R_{i_{|\theta|}}(\lambda)$ (see Remark 3.3).

We also use $R_{\theta}(\lambda)$ to denote the integral dominant weight corresponding to the weight diagram obtained in the following way. For each $a=1, \ldots,|\theta|$, let $t_{a}$ be the vertex where the $i_{a}$-th $\times$ of $D_{\lambda}$ is moved to by $R_{i_{a}}(\lambda)$. Delete from $D_{\lambda}$ all the $\times$ 's labeled by $i_{1}, i_{2}, \ldots, i_{|\theta|}$, and then place a $\times$ at each of the vertices $t_{1}, t_{2}, \ldots, t_{|\theta|}$. 
As an example, we observe that the third $\times$ in the weight diagram (3.3) can only be moved to vertex 11 , which is the move $R_{3}(\lambda)$. We indicate all right moves below

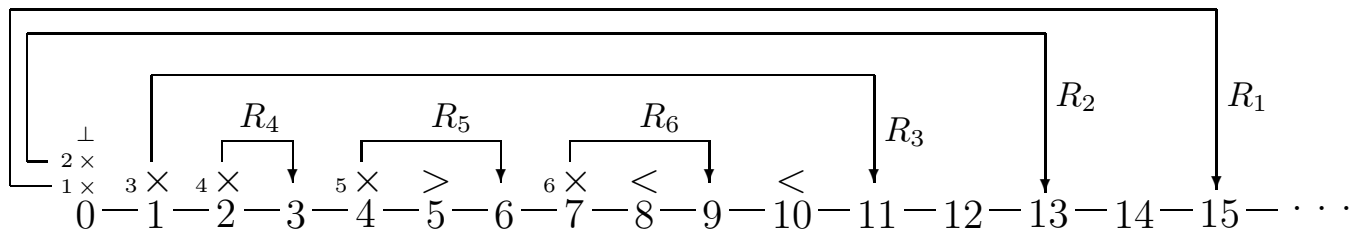

In particular, if $\theta=(1,1,1,1,1,1)$, we have

$$
R_{\theta}(\lambda)=(15,13,11,9,6,5,3,0,-3,-6,-8,-9,-10,-11,-13,-15) .
$$

In (3.4), we have written $R_{j}$ for $R_{j}(\lambda)$ and will do this in the future so long as there is no possibility of confusion.

Remark 3.3. We put $\lambda$ in the notations $R_{i_{a}}(\lambda)$ and $L_{i_{b}, j_{b}}(\lambda)$ (to be defined later) to emphasis the fact that the individual moves in a right path $R_{\theta}(\lambda)$ or left path $L_{\underline{i}, j}(\lambda)$ (to be defined later) are independently applied to the weight diagram $D_{\lambda}$ of $\lambda$, and not to the resulting diagram of previous moves.

Note from [2, Main Theorem] that the Euler module $E(\lambda)$ defined in (2.16) is in fact a true module if $\lambda \in \mathbb{Z}_{++}^{n}$. Using the notion of right paths, [2, Main Theorem] can be conveniently restated as follows.

Theorem 3.4. [2, Main Theorem] For any $\lambda, \mu \in \mathbb{Z}_{++}^{n}$, let $a_{\lambda \mu}:=[E(\lambda): L(\mu)]$ be the multiplicity of the composition factor $L(\mu)$ in $E(\lambda)$. Then

$$
a_{\lambda \mu}= \begin{cases}2^{\frac{z(\mu)-z(\lambda)}{2}} & \text { if } \lambda=R_{\theta}(\mu) \text { for some } \theta \in\{0,1\}^{\# \lambda}, \\ 0 & \text { otherwise. }\end{cases}
$$

Remark 3.5. If we delete from $D_{\lambda}$ all the vertices associateed with the symbols < and $>$ and relabel vertices, we obtain an $r$-fold atypical integral dominant weight $\lambda_{\text {red }} \in \mathbb{Z}_{++}^{2 r+\bar{z}(\lambda)}$. Then Theorem 3.4 depends only on $\lambda_{\text {red }}$ and $\mu_{\text {red }}$, i.e., $a_{\lambda \mu}=a_{\lambda_{\text {red }}, \mu_{\text {red }}}$.

3.3. Lowering operators. Given any $\mu \in \mathbb{Z}_{++}^{n}$, Theorem 3.4 provides a convenient algorithm for determining all $\lambda \in \mathbb{Z}_{++}^{n}$ such that $L(\mu)$ is a composition factor of the Euler module $E(\lambda)$. However, the theorem is unwieldy to use when one wants to determine the composition factors of a given Euler module $E(\lambda)$. We shall derive from Theorem 3.4 an algorithm which is more readily applicable for the latter task. For this, we need to introduce left moves and left paths following the general ideas of [23].

Definition 3.6. A left move (or lowering operator) is to move a $\times$ sitting at a vertex $x_{j}>0$ to the left

(1) either to an empty vertex $s>0$ if $d_{\lambda}\left(s, x_{j}\right)=0$ (in this case, if the number of $\times$ 's strictly between $s$ and $x_{j}$ is $k$, we let $i=j-k$ and denote the left move by $\left.L_{i j}(\lambda)\right)$

(2) or vertex 0 (and place it below $\perp$ in case there is a $\perp$ at vertex 0 ) if $d_{\lambda}\left(0, x_{j}\right) \in-2 \mathbb{Z}_{+}$(in this case we denote the left move by $L_{0 j}(\lambda)$ ).

We remark that a left move may move a $\times$ to different places, in contrast to a right move.

Definition 3.7. A left path (or simply a path) is the collection of left moves $L_{i_{1}, j_{1}}(\lambda)$, $\ldots, L_{i_{k}, j_{k}}(\lambda)$ (cf. Remark 3.3) satisfying the following conditions 
(i) $1 \leq j_{1}<\cdots<j_{k} \leq r$, and

(ii) for $1 \leq a<b \leq k$, if $i_{b} \leq j_{a}$ then $i_{b} \leq i_{a}$, and

(iii) for any $i_{b} \leq p<j_{b}$, if $\ell_{\lambda}\left(x_{p}, x_{j_{b}}\right) \leq 0$, then $p=j_{a}$ for some $a<b$.

Let $\underline{i}=\left(i_{1}, \ldots, i_{k}\right)$ and $\underline{j}=\left(j_{1}, \ldots, j_{k}\right)$, and denote by $L_{\underline{i}, j}(\lambda)$ the left path. If $k=0$, we use $L_{\emptyset}$ to denote this empty path. We shall also use $\bar{L}_{\underline{i}, \underline{j}}(\lambda)$ to denote the integral dominant weight corresponding to the weight diagram obtained in the following way. Let $s_{a}$ be the vertex where the $j_{a}$-th $\times$ of $\lambda$ is moved to by $L_{i_{a}, j_{a}}(\lambda)$ for $a=1,2, \ldots, k$. Delete from $\lambda$ the $\times$ 's labeled by $j_{1}, j_{2}, \ldots, j_{k}$ and then place a $\times$ at each of the vertices $s_{1}, s_{2}, \ldots, s_{k}$. Denote

$$
\Theta^{\lambda}=\text { the set of left paths of } \lambda .
$$

We also use $\Theta^{\lambda}$ to denote the set of integral dominant weights corresponding to the left paths of $\lambda$.

\section{Remark 3.8. In Definition 3.7,}

- condition (ii) means that in a left path no two moves are allowed to across each other (like $\sqrt{\sqrt{x}} \times$ );

- condition (iii) means that when a $\times$, say the $b$-th one, is moved to the left passing over another, say the $p$-th $\times($ like $\sqrt[\substack{\times \text {-th } \\ \text { b-th }}]{ }$ ), if the two $\times$ 's are too close in the sense that $\ell_{\lambda}\left(x_{p}, x_{j_{b}}\right) \leq 0$, then the $p$-th $\times$ must also be moved to the left whose destination is closer than that of the $b$-th $\times($ like $\sqrt{\sqrt{p \text {-th }} b \text {-th }}$.

We give some examples of left paths to illustrate the concept.

Example 3.9. If $\lambda$ is the weight in (3.3), one can easily obtain all the possible left paths for $D_{\lambda}$. There are only 4 paths in total, which are given by (cf. Remark 3.3)

$$
L_{\emptyset} \quad L_{55} \quad L_{66} \quad L_{56} .
$$

Example 3.10. Let $n=2 r$ and $\lambda=(2 r-1, \ldots, 3,1,-1,-3, \ldots,-2 r+1) \in \mathbb{Z}_{++}^{n}$. Then for any $i, j$ with $0 \leq j \leq i \leq r, j \neq 1$ and $i \geq 1$, we have the left move $L_{j i}$ (note that in this example we do not have $L_{1 i}$ as by Definition 3.6(1), $L_{j i}$ for $j \neq 0$ is to move to the left the $i$-th $\times$ to some empty vertex $s>0$; if we can allow $s=0$, then in fact $L_{1 i}$ coincides with $L_{0 i}$; this is why we require $s>0$ ). The total number of left paths is

$$
\# \Theta^{\lambda}=(r+1) C_{r}=\left(\begin{array}{c}
2 r \\
r
\end{array}\right),
$$

where $C_{r}:=\frac{1}{r+1}\left(\begin{array}{c}2 r \\ r\end{array}\right)$ is the $r$-th Calatan number.

To prove (3.8), let $\# \Theta^{\lambda}=x_{r}$. Clearly $x_{0}=1, x_{1}=2$. Assume $r \geq 2$. Considering the $r$-th $\times$, we have the following choices: (1) it is not moved; (2) $L_{r r}$, (3) $L_{0 r}$ and (4) $L_{i r}$ for $2 \leq i \leq r-1$.

Note that for the first 3 choices, the first $(r-1) \times$ 's can be moved to the left to any places so long as conditions in Definition 3.7 are satisfied by these $(r-1) \times$ 's. Thus there are in total $3 x_{r-1}$ left paths of this kind.

For choice (4), when $i$ is fixed, we have the following:

- the first $(i-1) \times$ 's can be moved to the left to any places so long as conditions in Definition 3.7 are satisfied by these $(i-1) \times$ 's. Thus we have $x_{i-1}$ choices.

- The $i$-th $\times$ cannot be moved to the left by condition (ii) in Definition 3.7 . 
- The remaining $(r-i-1) \times$ 's can be moved to the left to any places not passing over the $i$-th $\times$, so long as conditions in Definition 3.7 are satisfied by these $\times$ 's. Since vertex 0 is not involved in such left moves, in this case, conditions in Definition 3.7 are exactly the same as that of left paths for the general linear superalgebra $\mathfrak{g l}(m \mid n)$ [23, Definition 5.2]. Thus we have $C_{r-i}$ choices by [20, Lemma 3.12].

Therefore, we obtain

$$
x_{r}=3 x_{r-1}+\sum_{i=2}^{r-1} x_{i-1} C_{r-i} \text { for } r \geq 2 .
$$

From this, one can deduce (3.8) by induction on $r$.

Example 3.11. Let $n=2 r+1$ and $\lambda=(2 r, \ldots, 4,2,0,-2,-4, \ldots,-2 r) \in \mathbb{Z}_{++}^{n}$. Then for any $i, j$ with $0 \leq j \leq i \leq r$ and $i \geq 1$, we have the left move $L_{j i}$ (note that in this example, we have $L_{1 i}$ which is different from $L_{0 i}$, in contrast to the previous example). The total number of left paths is

$$
\# \Theta^{\lambda}=\frac{1}{2}(r+2) C_{r+1}=\frac{1}{2}\left(\begin{array}{c}
2 r+2 \\
r+1
\end{array}\right) .
$$

The proof is the same as that of the previous example with (3.9) replaced by $x_{r}=3 x_{r-1}+\sum_{i=1}^{r-1} x_{i-1} C_{r-i}$ for $r \geq 1$. From this we obtain (3.10).

3.4. Structure of Euler modules. The notion of left paths provides a useful way to describe the structure of Euler modules. We have the following result.

Theorem 3.12. Let $\lambda \in \mathbb{Z}_{++}^{n}$ be given. Then $L(\mu)$ with $\mu \in \mathbb{Z}_{++}^{n}$ is a composition factor of $E(\lambda)$ if and only if $\mu \in \Theta^{\lambda}$. In this case, the multiplicity is $a_{\lambda \mu}=2^{\frac{z(\mu)-z(\lambda)}{2}}$. In particular, the number of composition factors of $E(\lambda)$ (without counting the multiplicities) can vary from 1 to the maximal number $\frac{1}{2}\left(\begin{array}{c}2 r+2 \\ r+1\end{array}\right)$, where $r=\# \lambda$ is the degree of atypicality of $\lambda$.

Proof. The first and second statements are a reformulation of Theorem 3.4 in terms of left paths (cf. Definition 3.7), while the third statement follows from the first.

\section{Character and dimension formulae}

4.1. The $c$-relationship. Following [11, 21] we introduce the notion of $c$-relation between atypical roots of an integral dominant weight $\lambda$.

Definition 4.1. Let $\lambda$ be an $r$-fold atypical integral dominant weight with atypical roots (2.13). Suppose $1 \leq s \leq t \leq r$.

(1) We define the distance $d_{s t}(\lambda)$ of two atypical roots $\gamma_{s}, \gamma_{t}$ of $\lambda$ by $d_{s t}(\lambda)=0$ if the $s$-th and $t$-th $\times$ 's both sit at vertex 0 , and $d_{s t}(\lambda)=\ell_{\lambda}\left(s, x_{t}\right)$ otherwise, where $x_{t}$ is the vertex where the $t$-th $\times$ sits and $\ell_{\lambda}\left(s, x_{t}\right)$ is defined in Definition 3.2 .

(2) We say that two atypical roots $\gamma_{s}, \gamma_{t}$ of $\lambda$ are $c$-related [11, 21] (or connected [26]) if $d_{s t}(\lambda) \leq 0$, and are strongly c-related if $\gamma_{s}, \gamma_{p}$ are $c$-related for all $p$ such that $s \leq p \leq t$.

(3) As in [21], we let $\hat{c}_{s t}(\lambda)=1$ if $\gamma_{s}, \gamma_{t}$ are strongly $c$-related, and $\hat{c}_{s t}(\lambda)=0$ otherwise. 
Example 4.2. Let $\lambda$ be as in (3.2) with weight diagram (3.3) and right moves (3.4). One immediately sees that $\hat{c}_{s t}(\lambda)=1$ for $s=1,2,3$ and $s \leq t \leq 6$, and $\hat{c}_{s t}(\lambda)=0$ for $s=4,5,6$ and $s<t \leq 6$. In general if $1 \leq s<t \leq r, \hat{c}_{s t}(\lambda)=1$ if and only the right move $\tilde{R}_{s}(\lambda)$ passes over the $t$-th $\times$, where $\tilde{R}_{s}=R_{s}$ if $x_{s} \neq 0$ or there is a $\perp$ at vertex 0 , otherwise it is the move further to the right to the first empty vertex that is available (just image there is a $\perp$ at vertex 0 , then $\tilde{R}_{s}$ is the move $R_{s}$ ).

We need to understand $\hat{c}_{s t}(\lambda)$ better. Recall that in the weight diagram $D_{\lambda}$ of $\lambda$, there are $\left\lfloor\frac{z(\lambda)}{2}\right\rfloor$ many $\times$ 's (and in addition one $\perp$ if $\bar{z}(\lambda)=1$ ) located at vertex 0 . The following result can be easily verified.

Lemma 4.3. Let $\lambda$ be an r-fold atypical integral dominant weight as in (2.14) with atypical roots $\gamma_{p}=\varepsilon_{m_{p}}-\varepsilon_{n_{p}}$ for $1 \leq p \leq r$ as in (2.13). Then for $1 \leq s<t \leq r$,

$$
\hat{c}_{s t}(\lambda)=\left\{\begin{array}{l}
1 \quad \text { if } d_{s p} \leq 0 \text { for all } p \text { with } s<p \leq t \\
0 \quad \text { otherwise }
\end{array}\right.
$$

where $d_{s p}:=\lambda_{m_{p}}-\lambda_{m_{s}}+m_{p}-m_{s}+n_{s}-n_{p}+1-d_{s}$, and $d_{s}=\bar{z}(\lambda)$ if $s \leq\left\lfloor\frac{z(\lambda)}{2}\right\rfloor$ or 0 else.

4.2. Lexical weights. Let $\lambda$ be an $r$-fold atypical regular weight (not necessarily dominant) with the set $\Gamma_{\lambda}=\left\{\gamma_{1}, \ldots, \gamma_{r}\right\}$ of atypical roots ordered according to (2.13). As in [21], we define the atypical tuple of $\lambda$

$$
\operatorname{aty}_{\lambda}:=\left(\lambda_{m_{r}}, \ldots, \lambda_{m_{1}}\right) \in \mathbb{Z}^{r},
$$

and call $\lambda_{m_{s}}$ the $s$-th atypical entry of $\lambda$ for $1 \leq s \leq r$. We also define

$$
\operatorname{typ}_{\lambda} \in \mathbb{Z}^{n-2 r} \text { and } \bar{\lambda} \in \mathbb{Z}^{n}
$$

to be respectively the element obtained from $\lambda$ by deleting all entries $\lambda_{m_{s}}, \lambda_{n_{s}}$ and the element obtained by changing all entries $\lambda_{m_{s}}, \lambda_{n_{s}}$ to zero for $s=1, \ldots, r$. We call typ ${ }_{\lambda}$ the typical tuple of $\lambda$, whose all entries, called the typical entries of $\lambda$, have distinct absolute values by (2.10).

In the following, we fix an $r$-fold atypical integral dominant weight $\lambda$. Denote by $\mathcal{P}^{\lambda}$ the set of all integral weights $\mu$ such that $\Gamma_{\lambda}$ is a maximal set of orthogonal atypical roots of $\mu$ (with respect to the bilinear form (2.3) ) and $\bar{\mu}=\bar{\lambda}$. Hereafter, aty ${ }_{\mu}, \operatorname{typ}_{\mu}, \bar{\mu}$ are defined as in (4.2) and (4.3). Thus any $\mu \in \mathcal{P}^{\lambda}$ has the form

$$
\mu=\bar{\lambda}+\sum_{p=1}^{r} j_{p} \gamma_{p} \text { for some } j_{p} \in \mathbb{Z}
$$

If $\nu=\bar{\lambda}+\sum_{p=1}^{r} \ell_{p} \gamma_{p} \in \mathcal{P}^{\lambda}$, we define (cf. (2.1) )

$$
|\mu-\nu|=\left|\mu^{+}-\nu^{+}\right|=\sum_{p=1}^{r}\left(j_{p}-\ell_{p}\right) \quad(\text { called the relative level of } \nu \text { in } \mu) \text {. }
$$

Definition 4.4. (1) We call $\mu \in \mathcal{P}^{\lambda}$ lexical if its atypical tuple aty ${ }_{\mu}$ is lexical, where an element $a=\left(a_{r}, a_{r-1}, \ldots, a_{1}\right) \in \mathbb{Z}^{r}$ is called lexical if

$$
a_{r} \geq a_{r-1} \geq \ldots \geq a_{1}
$$

(2) Denote by $P_{\text {Lex }}^{\lambda}$ the subset of $\mathcal{P}^{\lambda}$ consisting of the lexical weights of $\mathcal{P}^{\lambda}$. 
We define the partial order $\preccurlyeq$ on $\mathcal{P}^{\lambda}$ (which is compatible with the total order $<$ defined in (2.9) ) for $\mu, \nu \in \mathcal{P}^{\lambda}$ by

$$
\nu \preccurlyeq \mu \quad \Longleftrightarrow \quad \operatorname{aty}_{\nu} \leq \operatorname{aty}_{\mu}
$$

where the partial order " $\leq$ " on $\mathbb{Z}^{r}$ is defined for $a=\left(a_{1}, \ldots, a_{r}\right), b=\left(b_{1}, \ldots, b_{r}\right) \in \mathbb{Z}^{r}$ by

$$
a \leq b \quad \Longleftrightarrow \quad a_{i} \leq b_{i} \text { for } 1 \leq i \leq r
$$

For any $\mu \in \mathcal{P}^{\lambda}$, we denote $\mathcal{P}^{\preccurlyeq \mu}:=\left\{\nu \in P_{\text {Lex }}^{\lambda} \mid \nu \preccurlyeq \mu\right.$ and $\left.\operatorname{aty}_{\nu} \in \mathbb{N}^{r}\right\}$, then

$$
\mathcal{P}^{\preccurlyeq \mu}=\left\{\bar{\mu}+\sum_{p=1}^{r} j_{p} \gamma_{p} \mid 0 \leq j_{1} \leq m_{1} \text { and } j_{p-1} \leq j_{p} \leq m_{p} \text { for } p=2, \ldots, r\right\},
$$

where $m_{p}=\min \left\{\mu_{p}, \mu_{p+1}, \ldots, \mu_{r}\right\}$.

4.3. The action of $\operatorname{Sym}_{r}$ on $\mathcal{P}^{\lambda}$. The symmetric group $\operatorname{Sym}_{r}$ of degree $r$ acts on $\mathbb{Z}^{r}$ by permuting entries. This action induces an action on $\mathcal{P}^{\lambda}$ given by

$$
\begin{gathered}
\sigma(\mu)=\left(\mu_{1}, \ldots, \mu_{m_{\sigma^{-1}(r)}}, \ldots, \mu_{i}, \ldots, \mu_{m_{\sigma^{-1}(1)}}, \ldots, \mu_{n_{\sigma^{-1}(1)}}, \ldots, \mu_{\zeta}, \ldots, \mu_{n_{\sigma^{-1}(r)}}, \ldots, \mu_{n}\right), \\
\begin{array}{c}
\text { atypical entries permuted } \\
\text { atypical entries permuted }
\end{array}
\end{gathered}
$$

for $\sigma \in \operatorname{Sym}_{r}$ and $\mu \in \mathcal{P}^{\lambda}$. With this action on $\mathcal{P}^{\lambda}$, the group $\mathrm{Sym}_{r}$ can be regarded as a subgroup of $W$, such that every element is of even parity. We will need the following

Definition 4.5. Let $\mu \in \mathcal{P}^{\lambda}, \sigma \in \mathrm{Sym}_{r}$, we define

$$
a_{\mu}^{\sigma}= \begin{cases}0 & \text { if } \hat{c}_{s t}(\mu)=1, s<t, \sigma^{-1}(s)>\sigma^{-1}(t) \text { for some } s, t, \\ 1 & \text { otherwise, }\end{cases}
$$

where $\hat{c}_{s t}(\mu)$ is defined in (4.1). Define

$$
\mathcal{S}^{\mu}=\left\{\sigma \in \operatorname{Sym}_{r} \mid a_{\mu}^{\sigma}=1\right\} .
$$

Namely, $\mathcal{S}^{\mu}$ is the subset of the symmetric group $\operatorname{Sym}_{r}$ consisting of permutations $\sigma$ which do not change the order of $s<t$ when the atypical roots $\gamma_{s}$ and $\gamma_{t}$ of $\mu$ are strongly $c$-related.

4.4. More on raising operators. First we generalise the notion of $r$-tuples of positive integers associated to weights as follows.

Definition 4.6. Let $\mu$ be a regular weight (cf. Definition 2.1(1)). Assume that $\mu$ is $r$-fold atypical with atypical roots $\gamma_{1}<\gamma_{2}<\cdots<\gamma_{r}$ as in (2.13). Let $w \in W$ be such that $\mu^{+}:=w(\lambda)$ is integral dominant. Then $\Gamma_{\mu^{+}}=w\left(\Gamma_{\mu}\right)$. Arrange the $r$ atypical roots of $\mu^{+}$as

$$
w\left(\gamma_{i_{1}}\right)<w\left(\gamma_{i_{2}}\right)<\cdots<w\left(\gamma_{i_{r}}\right) .
$$

We define the $r$-tuple of positive integers associated to $\mu$ to be the $r$-tuple $\left(k_{1}, \ldots, k_{r}\right)$ such that $\left(k_{i_{1}}, \ldots, k_{i_{r}}\right)$ is the $r$-tuple of positive integers associated to $\mu^{+}$.

For each $i(1 \leq i \leq r)$, we define a raising operator $\bar{R}_{i}$ on the regular weight $\lambda$ by

$$
\bar{R}_{i}(\lambda)=\lambda+k_{i} \gamma_{i}
$$

In particular, when $\lambda$ is integral dominant, by Definition 3.2 , we have $R_{i}(\lambda)=\left(\bar{R}_{i}(\lambda)\right)^{+}$ (recall from Definition 2.1(1) that in general $\mu^{+}$is the "dominant conjugate" of $\mu$ ) and

$$
R_{\theta}(\lambda)=\left(\bar{R}_{r}^{\theta_{r}} \bar{R}_{r-1}^{\theta_{r-1}} \cdots \bar{R}_{1}^{\theta_{1}}(\lambda)\right)^{+}=R_{j_{r}}^{\theta_{r}} R_{j_{r-1}}^{\theta_{r-1}} \cdots R_{j_{1}}^{\theta_{1}}(\lambda) \text { for } \theta \in\{0,1\}^{r},
$$

where $j_{p}=p-\#\left\{q<p \mid \theta_{q}=1, \hat{c}_{q p}(\lambda)=1\right\}$, and the product of operators $R_{i}$ is their composition. For example, $R_{i} R_{j}(\lambda)=R_{i}\left(R_{j}(\lambda)\right)$, which means applying first the right 
move of the $j$-th $\times$ of $\lambda$ then the right move of the $i$-th $\times$ to the resulting diagram. Now for any $\theta=\left(\theta_{1}, \ldots, \theta_{r}\right) \in \mathbb{N}^{r}$, we let $\bar{R}_{\theta}=\bar{R}_{1}^{\theta_{1}} \bar{R}_{2}^{\theta_{2}} \cdots \bar{R}_{r}^{\theta_{r}}$ and define the operator $R_{\theta}^{\prime}$ acting on integral dominant weights by

$$
R_{\theta}^{\prime}(\lambda)=\left(\bar{R}_{\theta}(\lambda)\right)^{+}=\left(\bar{R}_{1}^{\theta_{1}} \bar{R}_{2}^{\theta_{2}} \cdots \bar{R}_{r}^{\theta_{r}}(\lambda)\right)^{+}=R_{1}^{\theta_{1}} R_{2}^{\theta_{2}} \cdots R_{r}^{\theta_{r}}(\lambda)
$$

4.5. Character and dimension formulae. For any $\lambda, \mu \in \mathbb{Z}_{++}^{n}$, we define

$$
b_{\lambda \mu}=2^{\frac{z(\mu)-z(\lambda)}{2}} \sum_{\theta \in \mathbb{N}^{r}: \lambda=R_{\theta}^{\prime}(\mu)}(-1)^{|\theta|}, \quad \text { where }|\theta|=\sum_{i=1}^{r} \theta_{i} .
$$

Theorem 4.7. Order the elements of $\mathbb{Z}_{++}^{n}$ by (2.9), and let $A=\left(a_{\lambda \mu}\right)_{\lambda, \mu \in \mathbb{Z}_{++}^{n}}$, $B=\left(b_{\lambda \mu}\right)_{\lambda, \mu \in \mathbb{Z}_{++}^{n}}$, which are upper triangular matrices with diagonal entries being 1 . Then $B=A^{-1}$, and it follows that

$$
\operatorname{ch} L(\lambda)=\sum_{\mu \in \mathbb{Z}_{++}^{n}} b_{\lambda \mu} \operatorname{ch} E(\mu)=\sum_{\mu \in \mathbb{Z}_{++}^{n}} b_{\lambda \mu} 2^{\left\lfloor\frac{h(\mu)+1}{2}\right\rfloor} P_{\mu} .
$$

Proof. It suffices to prove that for $\mu<\lambda$ (cf. (2.9) or (4.7)),

$$
\sum_{\nu \in \mathbb{Z}_{++}^{n}} b_{\lambda \nu} a_{\nu \mu}=0
$$

Let $\Omega_{\mu}^{\lambda}=\left\{\left(\theta^{\prime}, \theta\right) \in \mathbb{N}^{r} \times\{0,1\}^{r} \mid \lambda=R_{\theta^{\prime}}^{\prime} R_{\theta}(\mu)\right\}$. Then clearly,

$$
\text { left-hand side of (4.19) }=2^{\frac{z(\mu)-z(\lambda)}{2}} \sum_{\left(\theta^{\prime}, \theta\right) \in \Omega_{\mu}^{\lambda}}(-1)^{\theta^{\prime}} \text {. }
$$

We shall define a bijective map ${ }^{\sim}: \Omega_{\mu}^{\lambda} \rightarrow \Omega_{\mu}^{\lambda},\left(\theta^{\prime}, \theta\right) \mapsto\left(\tilde{\theta}^{\prime}, \tilde{\theta}\right)$ satisfying

$$
\approx=\operatorname{id}_{\Omega_{\mu}^{\lambda}}, \quad\left|\tilde{\theta}^{\prime}\right|=\left|\theta^{\prime}\right| \pm 1, \quad|\tilde{\theta}|=|\theta| \mp 1 .
$$

For $\left(\theta^{\prime}, \theta\right) \in \Omega_{\mu}^{\lambda}$, let

$$
p=\max \left\{p \mid \theta_{p} \neq 0\right\}, \quad q=\max \left\{q \geq p \mid \hat{c}_{p q}(\mu)=0\right\}, \quad p^{\prime}=\max \left\{p^{\prime} \mid \theta_{p^{\prime}}^{\prime} \neq 0\right\} .
$$

(We take $p=q=0$ if $\theta=0$, and $p^{\prime}=0$ if $\theta^{\prime}=0$.) If $\theta=0$ (then $\theta^{\prime} \neq 0$ as $\lambda \neq \mu$ ) or $q<p^{\prime}$ (then by definition of $p$ and $q$, we have $\theta_{i}=0$ for $i>q$ ), we set $\tilde{\theta}^{\prime}=\theta^{\prime}-\epsilon_{p^{\prime}} \in \mathbb{N}^{r}, \tilde{\theta}=\theta+\epsilon_{p^{\prime}} \in\{0,1\}^{r}$, where in general $\epsilon_{i}=\left(\delta_{1 i}, \ldots, \delta_{r i}\right)$. Then $\lambda=R_{\tilde{\theta}^{\prime}}^{\prime} R_{\tilde{\theta}}(\mu)$ by (4.15) and (4.16) , i.e., $\left(\tilde{\theta}^{\prime}, \tilde{\theta}\right) \in \Omega_{\mu}^{\lambda}$.

Assume $\theta \neq 0$ and $p^{\prime} \leq q$. We denote

$$
\begin{aligned}
& \hat{\theta}^{\prime}=\left(\theta_{1}^{\prime}, \ldots, \theta_{p-1}^{\prime}, 0, \ldots, 0\right), \quad \bar{\theta}^{\prime}=\left(0, \ldots, 0, \theta_{p}^{\prime}, \ldots, \theta_{q-1}^{\prime}, 0, \ldots, 0\right), \\
& \hat{\theta}=\left(\theta_{1}, \ldots, \theta_{p-1}, 0, \ldots, 0\right), \quad \bar{\theta}=(\overbrace{0, \ldots, 0}^{p-1}, \theta_{p+1}, \ldots, \theta_{q}, 0, \ldots, 0) .
\end{aligned}
$$

Note from (4.15) that $R_{\theta}(\mu)=R_{\bar{\theta}} R_{p} R_{\hat{\theta}}(\mu)$, since after applying the right move $R_{p}$ to $R_{\hat{\theta}}(\mu)$, the $i$-th $\times$ for $p<i \leq q$ becomes the $(i-1)$-th $\times$ in the resulting diagram. Then by (4.16) and definitions of $p, q, p^{\prime}$ in (4.22), we have

$$
\lambda=R_{\theta^{\prime}}^{\prime} R_{\theta}(\mu)=R_{\hat{\theta}^{\prime}}^{\prime} R_{\bar{\theta}^{\prime}}^{\prime} R_{q}^{\theta_{q}^{\prime}} R_{\bar{\theta}} R_{p} R_{\hat{\theta}}(\mu)=R_{\hat{\theta}^{\prime}}^{\prime} R_{\bar{\theta}^{\prime}}^{\prime} R_{\bar{\theta}} R_{p}^{\theta_{q}^{\prime}+1} R_{\hat{\theta}}(\mu)
$$

Denote $\hat{\mu}=R_{\bar{\theta}} R_{p}^{\theta_{q}^{\prime}+1} R_{\hat{\theta}}(\mu), \bar{\lambda}=R_{\bar{\theta}^{\prime}}^{\prime}\left(R_{\bar{\theta}}(\hat{\mu})\right)$. Then $\lambda=R_{\hat{\theta}^{\prime}}^{\prime}(\bar{\lambda})$. If $\bar{\lambda}=\hat{\mu}$, i.e., $\bar{\theta}^{\prime}=\bar{\theta}=0$, then we set $\tilde{\theta}^{\prime}=\theta^{\prime}+\epsilon_{p} \in \mathbb{N}^{r}, \tilde{\theta}=\theta-\epsilon_{p} \in\{0,1\}^{r}$, and $\left(\tilde{\theta}^{\prime}, \tilde{\theta}\right) \in \Omega_{\mu}^{\lambda}$. 
Now assume $\hat{\mu}<\bar{\lambda}$. Then $\left(\bar{\theta}^{\prime}, \bar{\theta}\right) \in \Omega_{\hat{\mu}}^{\bar{\lambda}}$. By induction on the relative level $|\lambda-\mu|$ (cf. (4.5)), we can assume there exists a bijective map $\sim$ on $\Omega_{\hat{\mu}}^{\bar{\lambda}}$ satisfying (4.21). Now we take $\tilde{\theta}^{\prime}=\hat{\theta}^{\prime}+\tilde{\bar{\theta}}^{\prime}, \tilde{\theta}=\hat{\theta}+\epsilon_{p}+\tau(\tilde{\bar{\theta}})$, where $\tau(\tilde{\bar{\theta}})$ is obtained from $\tilde{\bar{\theta}}$ moving the 0 at the last entry to the first entry (note that $\theta=\hat{\theta}+\epsilon_{p}+\tau(\bar{\theta})$ by (4.23) ).

The above uniquely defines the bijection $\sim$ satisfying (4.21), from this we see that the right-hand of (4.20) is zero. Thus we have (4.19).

For any $\mu \in \mathcal{P}^{\lambda}$, we generalise the definition of Schur's P-function (2.17) by defining

$$
P_{\mu}:=\frac{1}{\# S_{\mu}} \sum_{w \in \operatorname{Sym}_{n}} w\left(e^{\mu} \prod_{\substack{1 \leq i<j \leq n \\\left(\mu_{i}, \mu_{j}\right) \neq(0,0)}} \frac{1+e^{\varepsilon_{j}-\varepsilon_{i}}}{1-e^{\varepsilon_{j}-\varepsilon_{i}}}\right),
$$

where $S_{\mu}$ is the stabilizer of $\lambda$ in $\operatorname{Sym}_{n}$. Note from Definition 2.1 that

$$
P_{\mu} \neq 0 \Longleftrightarrow \mu \text { is regular. }
$$

Also recall the definition of $\mathcal{P} \preccurlyeq \nu$ from (4.9). We have the following result.

Theorem 4.8. Let $L(\lambda)$ be the finite dimensional simple $\mathfrak{q}(n)$-module with highest weight $\lambda$.

(1) The formal character of $L(\lambda)$ is given by

$$
\operatorname{ch} L(\lambda)=\sum_{\sigma \in \operatorname{Sym}_{r}} \sum_{\mu \in \mathcal{P} \preccurlyeq \sigma(\lambda)}(-1)^{|\lambda-\mu|} 2^{\frac{z(\mu)-z(\lambda)}{2}+\left\lfloor\frac{h(\mu)+1}{2}\right\rfloor} a_{\lambda}^{\sigma} P_{\mu},
$$

where $a_{\lambda}^{\sigma}$ is defined by (4.11).

(2) The dimension of $L(\lambda)$ is given by

$$
\begin{aligned}
\operatorname{dim} L(\lambda)=\sum_{\sigma \in \operatorname{Sym}_{r}} & \sum_{\mu \in \mathcal{P} \preccurlyeq \sigma(\lambda)} \sum_{B \subset \Phi_{\overline{0}}^{+}}(-1)^{|\lambda-\mu|} 2^{\frac{z(\mu)-z(\lambda)}{2}+\left\lfloor\frac{h(\mu)+1}{2}\right\rfloor} \frac{a_{\lambda}^{\sigma}}{\# S_{\mu}} \\
& \times(-1)^{\left|B \cap \Phi_{\overline{0}}^{+}(\mu)\right|} \prod_{\alpha \in \Phi_{\overline{0}}^{+}} \frac{\left(\alpha, \mu+\sum_{\beta \in B} \beta+\rho_{\overline{0}}\right)}{\left(\alpha, \rho_{\overline{0}}\right)},
\end{aligned}
$$

where $\Phi_{\overline{0}}^{+}(\mu)=\left\{\varepsilon_{i}-\varepsilon_{j} \in \Phi_{\overline{0}}^{+} \mid i<j, \mu_{i}=\mu_{j}=0\right\}$.

Proof. Part (2) of the theorem can be proven in a similar way as in [21], thus we only give the details of the proof for part (1). By (4.12), we can assume $\sigma$ appearing in (4.27) is in $\mathcal{S}^{\lambda}$. By (4.26), we can assume $\mu$ appearing in (4.27) is regular and lexical. For convenience, without loss of generality, we may assume that the weight diagram

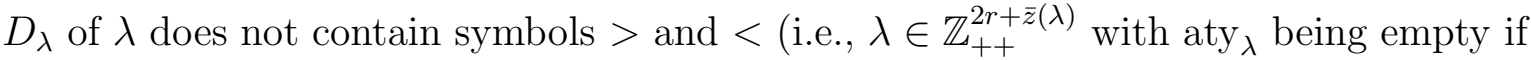
$\bar{z}(\lambda)=0$ or zero else, cf. Remark 3.5). In this case, regular and lexical weights coincide with integral dominant weights. Let $\mu \preccurlyeq \lambda$ be fixed. We want to prove that

$$
\Theta_{\mu}^{\lambda}:=\left\{\theta \in \mathbb{N}^{r} \mid R_{\theta}^{\prime}(\mu)=\lambda\right\},
$$

is in 1-1 correspondence with $\mathcal{S}^{\lambda}$. If so, then (4.27) follows from the claim and (4.17), by noting that $(-1)^{|\theta|}=(-1)^{|\lambda-\mu|}$ for $\theta \in \Theta_{\mu}^{\lambda}$ (which can be easily proven).

Let $\theta \in \Theta_{\mu}^{\lambda}$, we define the unique element $\sigma_{\theta} \in \operatorname{Sym}_{r}$ such that for each $p=1, \ldots, r$, the $p$-th $\times$ of $D_{\mu}$ is moved to the $\sigma_{\theta}(p)$-th $\times$ of $D_{\lambda}$ by the raising operator $R_{\theta}^{\prime}$. Note that if $1 \leq s<t \leq r$ such that $\bar{c}_{s t}(\lambda)=1$, then the $t$-th $\times$ of $D_{\lambda}$ has to be reached by $\sigma_{\theta}^{-1}(t)$-th $\times$ of $D_{\mu}$ earlier than the $s$-th $\times$ of $D_{\lambda}$ by $\sigma_{\theta}^{-1}(s)$-th $\times$ of $D_{\mu}$ by the rasing 
operator $R_{\theta}^{\prime}$ (for example, assume $\lambda$ is as in (3.4), if the 3 -rd $\times$ of $D_{\lambda}$ is obtained by some raising operator from some $\times$ of some $D_{\mu}$ before the 4 -th $\times$, then there is no way that the 4 -th $\times$ of $D_{\lambda}$ can be obtained by some rasing operator from any $\times$ of any $D_{\mu}$ ). Thus by definition of $R_{\theta}^{\prime}$ in (4.16), we have $\sigma_{\theta}^{-1}(t)>\sigma_{\theta}^{-1}(s)$. This proves $\sigma_{\theta} \in \mathcal{S}^{\lambda}$. Now one can easily see that the map $\Theta_{\mu}^{\lambda} \rightarrow \mathcal{S}^{\lambda}: \theta \mapsto \sigma_{\theta}$ is a bijection. This proves the claim and the theorem.

4.6. A corollary. We can manipulate Theorem 4.8 to express $\operatorname{ch} L(\lambda)$ and $\operatorname{dim} L(\lambda)$ in more convenient forms. For any $r$-fold atypical integral dominant weight $\lambda$ with atypical roots $\gamma_{1}<\ldots<\gamma_{r}$ as in (2.13), we define the normal cone with vertex $\lambda$ (cf. (4.2), (4.3)) in analogy to [21]:

$$
\begin{aligned}
\mathcal{C}_{\lambda}^{\text {Norm }} & =\left\{\mu:=\lambda-\sum_{s=1}^{r} i_{s} \gamma_{s} \mid i_{s} \geq 0, \text { aty }_{\mu} \in \mathbb{N}^{r}\right\} \\
& =\left\{\bar{\lambda}+\sum_{p=1}^{r} j_{p} \gamma_{p} \mid 0 \leq j_{p} \leq \lambda_{m_{p}} \text { for } p=1, \ldots, r\right\} .
\end{aligned}
$$

We also define $\mathcal{C}_{\lambda}^{\text {Trun }}$, which was referred to as the truncated cone with vertex $\lambda$ in [25], to be the subset of $\mathcal{C}_{\lambda}^{\text {Norm }}$ consisting of weights $\mu$ such that the $s$-th entry of the atypical tuple aty $_{\mu}$ is smaller than or equal to the $t$-th entry of aty ${ }_{\mu}$ when the atypical roots $\gamma_{s}, \gamma_{t}$ of $\lambda($ not $\mu$ ) are strongly $c$-related for $s<t$. Namely

$$
\mathcal{C}_{\lambda}^{\text {Trun }}=\left\{\mu \in \mathcal{C}_{\lambda}^{\text {Norm }} \mid \mu_{m_{s}} \leq \mu_{m_{t}} \text { if } \widehat{c}_{s, t}(\lambda)=1 \text { for } s<t\right\} .
$$

For each $\mu \in \mathcal{C}_{\lambda}^{\text {Trun }}$, we define $b_{\mu}^{\lambda} \in \mathbb{C}$ as follows. We set $b_{\mu}^{\lambda}=0$ if $\mu$ is not regular and $b_{\mu}^{\lambda}=b_{\mu_{\text {red }}}^{\lambda_{\text {red }}}$ otherwise, where $\lambda_{\text {red }}$ is defined as in Remark 3.5 and $\mu_{\text {red }}$ is defined as follows: First take $\sigma \in \operatorname{Sym}_{n}$ such that $\nu:=\sigma(\mu)$ is dominant. Then we have $\nu_{\text {red }}$. Take $\mu_{\text {red }}=\sigma^{-1}\left(\nu_{\text {red }}\right)$ (note that $\sigma^{-1}$ induces an action on atypical entries of $\mu$, thus induces an action on $\nu_{\text {red }}$ ). Thus we can suppose $\lambda=\lambda_{\text {red }}$ and $\mu=\mu_{\text {red }}$. Note that in this case, the $p$-th atypical root of $\mu$ is $\gamma_{p}=\varepsilon_{r-p+1}-\varepsilon_{n-r+p}$, and $\lambda_{p}=\lambda_{m_{r-p+1}}$. If $\mu=0$, we define (cf. (4.29) )

$$
b_{0}^{\lambda}:=\# \Theta_{0}^{\lambda}=\frac{\# \mathcal{S}^{\lambda}}{(r+\bar{z}(\lambda)) !} d_{\lambda_{1}+s_{1}, r} d_{\lambda_{2}+s_{2}, r-1} \cdots d_{\lambda_{r}+s_{r}, 1},
$$

where $d_{i, k}=\min \left\{\left\lfloor\frac{i+1-\bar{z}(\lambda)}{2}\right\rfloor, k\right\}, s_{p}$ is the number of $q<p$ such that $\hat{c}_{p q}(\lambda)=1$, and where the last equality follows from combinatorics. Suppose $\mu \neq 0$. Set $i>0$ to be the largest such that $\mu_{i}>0$. Let $\mu^{\prime} \in \mathbb{Z}^{n-2}$ be obtained from $\mu$ by deleting its $i$-th and $(m+1-i)$-th entries, and let $\lambda^{\prime} \in \mathbb{Z}_{+}^{n-2}$ be obtained from $\lambda$ by deleting its $i$-th and $(n+1-i)$-th entries and subtracting its $j$-th entry by 2 and adding its $(n+1-j)$-th entry by 2 for all $j<i$. Then $b_{\mu}^{\lambda}=b_{\mu^{\prime}}^{\lambda^{\prime}}$. This defines all $b_{\mu}^{\lambda}$.

Note that $b_{\mu}^{\lambda}$ can also be described in the following way. For any fixed $\lambda$, each regular $\mu \in \mathcal{C}_{\lambda}^{\text {Trun }}$ uniquely corresponds to a weight diagram $D_{\mu}$ which is simply the diagram $D_{\mu^{+}}$(cf. (2.1)) but with the $i_{p^{-}}$th $\times$of $D_{\mu^{+}}$relabeled as the $p$-th $\times$ of $D_{\mu}$ (where the $p$-th atypical root of $\mu$ corresponds to the $i_{p}$-th atypical root of $\mu^{+}$, cf. (4.13) in Definition 4.6) for each $p=1, \ldots, r$. Now similar to Remark 3.5, delete all vertices of $D_{\lambda}$ which associate with symbols $<,>$, and further delete the vertex associated with $i_{p}$-th $\times$ of $D_{\lambda}$ such that the $p$-th $\times$ of $D_{\mu}$ is not located at vertex 0 for all $p$. Relabeling the remaining vertices, we obtain a dominant weight, denoted $\nu$. Then $b_{\mu}^{\lambda}=b_{0}^{\nu}$ (which is defined as in (4.32) ). 
Corollary 4.9. The formal character and dimension of the finite dimensional irreducible $\mathfrak{q}(n)$-module $L(\lambda)$ are respectively given by

$$
\begin{aligned}
\operatorname{ch} L_{\lambda}= & \sum_{\mu \in \mathcal{C}_{\lambda}^{\operatorname{Trun}}}(-1)^{|\lambda-\mu|} 2^{\frac{z(\mu)-z(\lambda)}{2}+\left\lfloor\frac{h(\mu)+1}{2}\right\rfloor} b_{\mu}^{\lambda} P_{\mu}, \\
\operatorname{dim} L(\lambda)= & \sum_{\mu \in \mathcal{C}_{\lambda}^{\operatorname{Trun}}} \sum_{B \subset \Phi_{\overline{0}}^{+}}(-1)^{|\lambda-\mu|} 2^{\frac{z(\mu)-z(\lambda)}{2}+\left\lfloor\frac{h(\mu)+1}{2}\right\rfloor} \frac{b_{\mu}^{\lambda}}{\# S_{\mu}} \\
& \times(-1)^{\left|B \cap \Phi_{\overline{0}}^{+}(\mu)\right|} \prod_{\alpha \in \Phi_{\overline{0}}^{+}} \frac{\left(\alpha, \mu+\sum_{\beta \in B} \beta+\rho_{\overline{0}}\right)}{\left(\alpha, \rho_{\overline{0}}\right)} .
\end{aligned}
$$

Proof. The result follows from the definition of $b_{\mu}^{\lambda}$ and Theorem 4.8 .

\section{REFERENCES}

[1] J. Brundan, Kazhdan-Lusztig polynomials and character formulae for the Lie superalgebra gl $(m \mid n)$. J. Amer. Math. Soc. 16 (2003) 185-231.

[2] J. Brundan, Kazhdan-Lusztig polynomials and character formulae for the Lie superalgebra $\mathfrak{q}(n)$, Adv. Math. 182 (2004) 28-77.

[3] J. Brundan, A. Kleshchev, Modular representations of the supergroup Q(n). J. Algebra 260 (2003), no. 1, 64-98.

[4] J. Brundan, C. Stroppel, Highest weight categories arising from Khovanov's diagram algebra I, II, III, IV. Moscow Math. J. 11 (2011) 685-722; Transform. Groups 15 (2010) 1-45; Represent. Theory 15 (2011) 170-243; J. Eur. Math. Soc. 14 (2012) 373-419.

[5] Cheng, Shun-Jen; Lam, Ngau. Irreducible characters of general linear superalgebra and super duality. Comm. Math. Phys. 298 (2010) 645-672.

[6] Cheng, Shun-Jen; Lam, Ngau; Wang, Weiqiang. Super duality and irreducible characters of orthosymplectic Lie superalgebras. Invent. Math. 183 (2011) 189-224.

[7] Cheng, Shun-Jen; Wang, Weiqiang; Zhang, R. B. Super duality and Kazhdan-Lusztig polynomials. Trans. Amer. Math. Soc. 360 (2008), no. 11, 5883-5924.

[8] S.-J. Cheng, R.B. Zhang, An analogue of Kostant's u-cohomology formula for the general linear superalgebra. International Math Research Notices 1 (2004) 31-53.

[9] S.-J. Cheng, R.B. Zhang, Howe duality and combinatorial character formula for orthosymplectic Lie superalgebras. Adv. Math. 182 (2004), no. 1, $124-172$.

[10] C. Gruson, V. Serganova, Cohomology of generalized supergrassmannians and character formulae for basic classical Lie superalgebras. Proc. London Math. Soc. 101 (2010) 852-892.

[11] J.W.B. Hughes, R.C. King, J. van der Jeugt, On the composition factors of Kac modules for the Lie superalgebras $s l(m \mid n)$. J. Math. Phys. 33 (1992) 470-491.

[12] V.G. Kac, Lie superalgebras. Adv. Math. 26 (1977) 8-96.

[13] V.G. Kac, Representations of classical Lie superalgebras, Lect. Notes Math. 676 (1978) 597-626.

[14] I. Penkov, V. Serganova, Cohomology of $G / P$ for classical complex Lie supergroups $G$ and characters of some atypical $G$-supermodules. Ann. Inst. Fourier 39 (1989) 845-873.

[15] I. Penkov, V. Serganova, Characters of irreducible $G$-modules and cohomology of $G / P$ for the Lie supergroup $G=Q(N)$. J. Math. Sci. 84 (5) (1997) 1382-1412.

[16] I. Penkov, V. Serganova, Characters of finite dimensional irreducible $\mathfrak{q}(n)$-supermodules, Lett. Math. Phys. 40 (2) (1997) 147-158.

[17] V. Serganova, Kazhdan-Lusztig polynomials and character formula for the Lie superalgebra gl $(m \mid n)$. Selecta Math. 2 (1996) 607-654.

[18] V. Serganova, Characters of irreducible representations of simple Lie superalgebras, Proceedings of the International Congress of Mathematicians 1998, Berlin, Vol. II, Documenta Mathematica, Journal der Deutschen Mathematiker-Vereinigung, pp. 583-593.

[19] Scheunert, M. The theory of Lie superalgebras. An introduction. Lecture Notes in Mathematics, 716. S pringer, Berlin, 1979. 
[20] Y. Su, Composition factors of Kac modules for the general linear Lie superalgebras. Math. Z. 252 (2006) 731-754.

[21] Y. Su, R.B. Zhang, Character and dimension formulae for general linear superalgebra. Adv. Math. 211 (2007) 1-33.

[22] Y. Su, R.B. Zhang, Generalised Verma modules for the orthosymplectic Lie superalgebra osp $(k \mid 2)$. J. Algebra 357 (2012) 94-115.

[23] Y. Su, R.B. Zhang, Generalised Jantzen filtration of Lie superalgebras I. J. Eur. Math. Soc. 14 (2012) 1103-1133.

[24] Y. Su, R.B. Zhang, Generalised Jantzen filtration of Lie superalgebras II: the exceptional cases. arXiv:1303.4797.

[25] J. van der Jeugt, J.W.B. Hughes, R.C. King, J. Thierry-Mieg, Character formulas for irreducible modules of the Lie superalgebras $\operatorname{sl}(m \mid n)$. J. Math. Phys. 31 (1990) 2278-2304.

[26] J. van der Jeugt, R.B. Zhang, Characters and composition factor multiplicities for the Lie superalgebra $g l(m \mid n)$. Lett. Math. Phys. 47 (1999) 49-61.

Department of Mathematics, Tongui University, Shanghai 200092, China

E-mail address: ycsu@tongji.edu.cn

School of Mathematics and Statistics, University of Sydney, NSW 2006, Australia

E-mail address: rzhang@maths.usyd.edu.au 